\title{
Knockdown of Histone Methyltransferase WHSC1 Induces Apoptosis and Inhibits Cell Proliferation and Tumorigenesis in Salivary Adenoid Cystic Carcinoma
}

\author{
CHAO LIU ${ }^{1,2^{*}}$, YU-HANG JIANG $^{3 *}$, ZE-LIANG ZHAO ${ }^{1}$, HAI-WEI WU ${ }^{1,2}$, LING ZHANG $^{1}$, \\ ZHIJIAN YANG ${ }^{4,5}$, ROBERT M. HOFFMAN ${ }^{4}$ and JIA-WEI ZHENG ${ }^{1 *}$ \\ ${ }^{1}$ Department of Oromaxillofacial Head and Neck Oncology, Shanghai Ninth People's Hospital, \\ College of Stomatology, Shanghai Jiao Tong University School of Medicine, Shanghai, P.R. China; \\ ${ }^{2}$ Department of Oral and Maxillofacial Surgery, \\ Affiliated Shandong Provincial Hospital of Shandong University, Shandong, P.R. China; \\ ${ }^{3}$ Department of Orthopedic Surgery, Shanghai Ninth People's Hospital, Shanghai, P.R. China; \\ ${ }^{4}$ AntiCancer, Inc., San Diego, CA, U.S.A.; \\ ${ }^{5}$ Origin Biosciences Inc., Nanjing, P.R. China
}

\begin{abstract}
Background/Aim: Salivary adenoid cystic carcinoma (SACC) is the most common malignancy of the salivary gland with a poor prognosis and survival. The present study aimed to investigate the role of histone methyltransferase WHSC1 in SACC. Materials and Methods: Human SACC specimens were evaluated for WHSCl expression by RT-PCR and immunohistochemistry. The effects of WHSCl knockdown on SACC cells proliferation, cell cycle, clone and tumorsphere formation, and apoptosis as well as on the expression of related genes were examined. A xenograft mouse model of SACC was used to evaluate the in vivo effects of WHSCl knockdown on SACC tumorigenesis. Results: WHSC1 expression was up-regulated in human SACC tissues $(p<0.01)$. WHSC1 knockdown in SACC cells significantly inhibited cell proliferation, clone and tumorsphere formation $(p<0.05)$. Cell distribution at the $S$ and $G_{2} / M$ phases was significantly reduced by WHSCl knockdown $(p<0.05)$. WHSCl knockdown significantly increased apoptosis of SACC cells $(p<0.05)$. $c$-Myc, survivin, $B c l-2$ and cyclin B1 genes were significantly down-regulated
\end{abstract}

This article is freely accessible online.

*These Authors contributed equally to this study.

Correspondence to: Jia-Wei Zheng, Department of Oromaxillofacial Head and Neck Oncology, Shanghai Ninth People's Hospital, College of Stomatology, Shanghai Jiao Tong University School of Medicine, Shanghai 200011, P.R. China. Tel: +86 13817999402, e-mail: davidzhengjw@sjtu.edu.cn

Key Words: WHSC1, salivary adenoid cystic carcinoma, H3K36 dimethylation, cell proliferation, apoptosis. by WHSC1 knockdown cells ( $p<0.05)$. WHSC1 knockdown significantly reduced $\mathrm{H} 3 \mathrm{~K} 36 \mathrm{me} 2$ modification of the MYC gene promoter in SACC cells and tumorigenesis of SACC cells in vivo $(p<0.05)$. Conclusion: Knockdown of WHSC1 inhibited cell proliferation, induced apoptosis and affected tumorigenesis in SACC.

Salivary adenoid cystic carcinoma (SACC) arises from the secretory epithelial cells of the salivary glands (1) and is the most common malignancy of the salivary glands, accounting for about $25-50 \%$ of malignant tumors in the major or the minor salivary glands, respectively (2-4). SACC demonstrates unique characteristics that lead to poor prognosis and low overall survival rates (5). Recent molecular pathology findings suggest that genetic translocation and/or overexpression of oncoproteins is important in salivary gland tumorigenesis and diagnosis. Studies have found that the recurrent MYB-NFIB gene fusion is the main genomic hallmark of $\operatorname{SACC}(6,7)$, However, further molecular genetic studies are needed to identify biomarkers as well as therapeutic targets or prognostic factors of ACC.

Histone methylation modification is an important process involved in the regulation of oncogenic gene expression and is controlled by histone methyltransferases and demethylases. WHSC1 (also known as NSD2) is a histone methyltransferase that mediates di-methylation of histone 3 lysine 36 (H3K36me2). H3K36 methylation has been found to be associated with transcriptional activation in many cancers 8). Increased expression of WHSC1 was found in many solid tumors, such as multiple myeloma, prostate cancer, breast cancer and head and neck cancer (8-10). In vitro studies indicated that WHSC1 modulates Ras-related 
Table I. Primers used for real-time quantitative PCR.

\begin{tabular}{lll}
\hline Gene name & Forward & Reverse \\
\hline$W H S C 1$ & CCCACCATACAAGCACAT & TCAGACACTCCGAATCAAA \\
GAPDH & TCTGATTTGGTCGTATTGGG & GGAAGATGGTGATGGGATT \\
$B C L-2$ & GTGGCCTTCTTTGAGTTCG & CATCCCAGCCTCCGTTAT \\
$B A X$ & GCGGAGTTCACAGCTCTATAC & AAAAGGCCCTACAGTTACCA \\
$B A D$ & CCCAGAGTTTGAGCCGAGTG & CCCATCCCTTCGTCGTCCT \\
$C C N B 1$ & CCCGAGAGGTCTTTTCCGAG & CCAGCCCATGATGGTTCTGAT \\
$B I R C 5$ & GCCGAGTGGAAACTTTTGTCG & GGCAGCGTGTACTTATCCTTCT \\
$M Y C$ & ACAGCGTCTGCTCCACCT & CTGCGTAGTTGTGCTGATGT \\
\hline
\end{tabular}

Table II. Primers used for ChIP-qPCR analysis.

\begin{tabular}{lll}
\hline Gene name & Forward & Reverse \\
\hline CHIP- $M Y C-P 1$ & TATCTTGGAGGCTGGTGT & TTAGCAGAGCGTAGTGGC \\
CHIP- $M Y C-P 2$ & GGTCTCCACCTTGCCTC & CCAATAATCCAGTGTCCCTA \\
CHIP $-M Y C-P 3$ & CGGACTAGGTGTTCAGGT & CCGTGTATGTGGGAGTGT \\
CHIP- $M Y C-P 4$ & GGTGCCTGTCCTCTTACT & AGGAGGGCTCTTCTTTC \\
CHIP $-M Y C-P 5$ & CCAGGAGGAGGAGAAAGG & GGATAAATGAAGGCAGGAC \\
CHIP- $M Y C-P 6$ & TAAGGCAACGATCCCATC & GACTTCTGCGAATACCGG \\
CHIP $-M Y C-P 7$ & TGTATGCCCTGCTTTCAC & CGGTTATCGTACCCTGTTC \\
CHIP- $M Y C-P 8$ & CCACAGGGCGATGTGTC & GAGTGGGATGCGGGAGAT
\end{tabular}

C3 botulinum toxin substrate 1 (Rac1) transcription, twist family transcription factor 1 (TWIST1), and nuclear factor $\mathrm{KB}(\mathrm{NF}-\mathrm{kB})$ to promote tumorigenesis and metastasis (11, 12). However, the role of WHSC1 in SACC remains unclear.

In the present study, WHSC1 was found to be up-regulated in human SACC. The effects of WHSC1 knockdown in SACC cells on cell proliferation, apoptosis and tumorigenesis were investigated.

\section{Materials and Methods}

Patients and specimens. A total of 23 salivary adenoid cystic carcinomas and 23 matched paracarcinoma normal salivary gland specimens were obtained from patients who underwent surgery between 2002 and 2014 at the Affiliated Shandong Provincial Hospital of Shandong University. Diagnosis was confirmed histologically by experienced pathologists. The study was approved by Research Ethics Committee of Affiliated Shandong Provincial Hospital of Shandong University (Approval number: 2018-230) and written informed consent was obtained from all patients.

Immunohistochemistry (IHC). IHC was performed on $5 \mu$ m-thick formalin fixed paraffin embedded slides. After deparafinization, rehydration, antigen retrieval, and endogenous peroxidase blockage. Samples were then incubated with the following antibodies: antiWHSC1 (ab75359, Abcam, Cambridge, UK), anti-cleaved caspase3 (9661, Cell Signaling Technology, Boston, USA), anti-Ki67
(GB13030-2, Servicebio, Wuhan, P.R. China) at $4^{\circ} \mathrm{C}$ for $8-10 \mathrm{~h}$. The sections were then incubated with secondary antibody (7074, Cell Signaling Technology, Boston, MA, USA) for $30 \mathrm{~min}$ at room temperature. Color development was performed with 3'diaminobenzidine (DAB) (K5007, DAKO, Copenhagen, DK). Nuclei were lightly counter stained with hematoxylin. The slides were viewed using a microscope (DM 4000B, Leica, Wetzlar, Germany) and positive cells were recognized by the appearance of brown staining. Expression levels were assessed by staining indexes using a 10-point quantification method (13). Scores of 0-5 indicate low expression, while scores of 6-10 indicate high expression.

Cell culture. Human salivary adenoid cystic carcinoma cell lines SACC-83 and SACC-LM were purchased from American Type Culture Collection. Cells were grown in DMEM (Hyclone, UT, USA) with $10 \%$ FBS (Gibco, CA, USA) and antibiotics (penicillin $100 \mathrm{U} / \mathrm{ml}$, streptomycin $100 \mu \mathrm{g} / \mathrm{ml}$ ) at $37^{\circ} \mathrm{C}$ in a humidified atmosphere with $5 \% \mathrm{CO}_{2}$.

shRNAs transfection. Two WHSC1-specific short hairpin RNAs (WHSC1-KD1 and WHSC1-KD2 shRNA) were designed and cloned into the pLVX-shRNA1 plasmid. A scramble nonsense sequence was used as a negative control. The plasmids were packaged into lentivirus using a three-plasmid system, pLVXshRNA1 with targeted sequence, psPAX2 and pMD2G. SACC-83 and SACC-LM cells were transfected and screened with $2 \mathrm{mg} / \mathrm{ml}$ puromycin for 2 weeks. The WHSC1-KD1 shRNA sequence was TGCCAATAACACGTCCACT, and the WHSC1-KD2 shRNA sequence was CCCTTCGCAGTGTTTGTCT. 
A

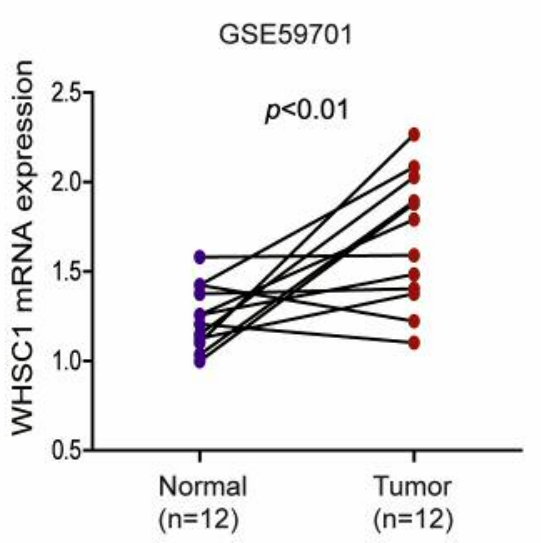

C

Normal

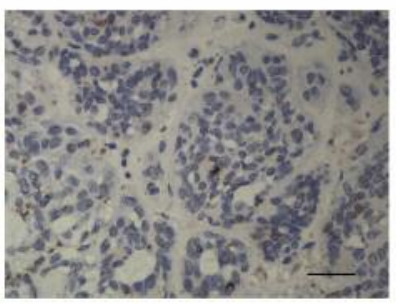

WHSC1
B

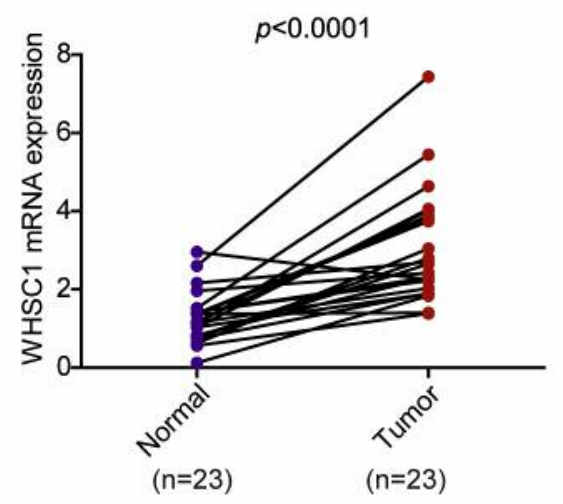

D

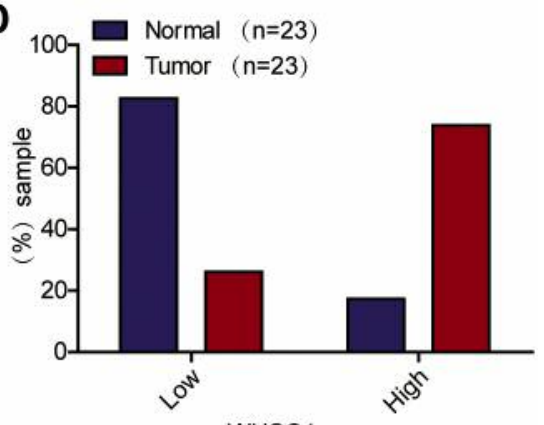

WHSC 1

Figure 1. Up-regulation of histone methyltransferase WHSC1 in human SACC. (A) WHSC1 up-regulation was found in the GSE59701 cohort. (B) WHSC1 mRNA levels in the tumor and matched normal tissues (paired t-test). (C and D) Immunohistochemical determination of WHSC1 expression in SACC specimens and normal salivary glands. ${ }^{*} p<0.01$, when compared to normal tissues.

Cell proliferation assay. Cell proliferation was measured using the CCK8 assay. The cells $\left(5 \times 10^{3}\right)$ were transferred to a 96 -well plate in RPMI 1640 containing 10\% FBS. After 24, 48 and $72 \mathrm{~h}$ of incubation, CCK8 reagent (1:10 dilution) was added to each well for $2 \mathrm{~h}$. The optical density (OD) at a wavelength of $450 \mathrm{~nm}$ was determined by a microplate reader (Bio-Rad, CA, USA). Proliferation curves were plotted for each of the cell groups.

Cloning efficiency assay. 1000 cells were added in each well of 6well plate, and the resulting colonies were fixed, and stained with $0.1 \%$ crystal violet at day 7 and colony number was counted.

Tumorsphere formation. Single-cell suspensions were added to ultralow-attachment plates in defined medium at a density of 1000 cells/well. The defined serum-free medium contained DMEM/F-12 supplemented with B27 (1:50) supplement, EGF (20 ng/ml), and FGF $(10 \mathrm{ng} / \mathrm{ml})$. Seven days after continuous cultivation, the number of spheres was recorded under a microscope. One-half volume of the medium was added every second day. The images were collected on day 7th with an inverted microscope, only spheres larger than $100 \mu \mathrm{m}$ were quantitated.

Apoptosis assay. The percentage of cells undergoing apoptosis was evaluated by flow cytometry (Beckman Gallios, Brea, CA, USA). After incubating with cisplatin for $48 \mathrm{~h}$, cells were harvested, washed and resuspended in $100 \mu \mathrm{l}$ staining buffer added with $1 \mu \mathrm{l}$
7-AAD (559925, BD Biosciences, NJ, USA) and $5 \mu$ l Annexin-VAPC (556421, BD Biosciences).

Cell cycle analysis. 5-ethynyl-2 deoxyuridine (EdU) assay was performed according to the manufacturer's instructions $(\mathrm{C} 0071 \mathrm{~L}$, Beyotime, Shanghai, P.R. China), SACC-83 and SACC-LM cells were incubated with EdU for $2 \mathrm{~h}$.

RNA extraction and real-time quantitative PCR. Total RNA was isolated from cells using TRIzol Reagent (15596-018, Invitrogen, Carlsbad, CA, USA). $1 \mu \mathrm{g}$ total RNA was reversely transcribed using the TaKaRa RT-PCR kit (Takara, Shiga, Japan) according to the manufacturer's instructions. followed by PCR amplification with specific primers (Table I). Data were analyzed by the relative standard curve method and normalized to $G A P D H$ expression. Relative RNA expression in the cells was calculated using the $2^{-\Delta \Delta \mathrm{Ct}}$ method.

Western blotting. Protein cell extracts were obtained with RIPA buffer (Roche Diagnostics, Indianapolis, IN, USA). $30 \mu \mathrm{g}$ of protein lysate was separated by $10 \%$ SDS-PAGE gel electrophoresis and electrophoretically transferred to a polyvinylidene fluoride (PVDF) membrane (Millipore, Bedford, MA, USA). The membranes were incubated with $10 \%$ nonfat milk solution for blocking non-specific binding and then with anti-c-Myc (9402, Cell Signaling Technology, Boston, MA, USA), anti-WHSC1 (ab75359, Abcam, Cambridge, UK), anti-Bcl-2 (4223S, Cell Signaling Technology), anti-cyclin B1 
A

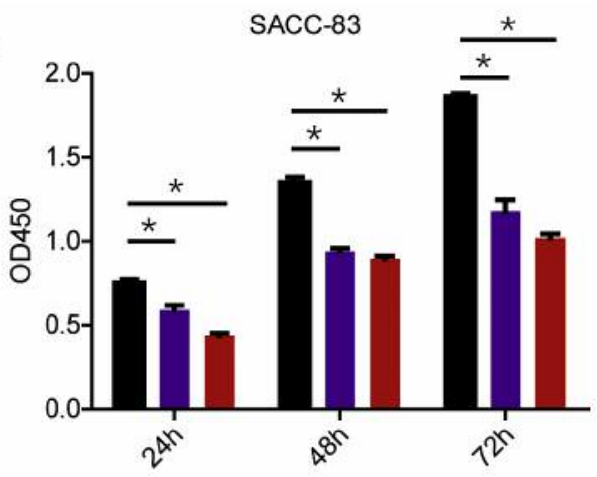

C

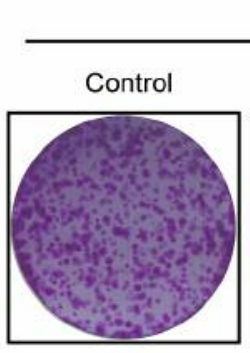

SACC-83
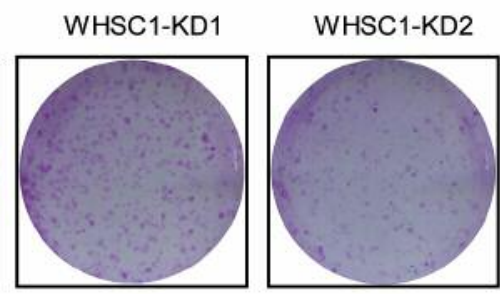

SACC-LM

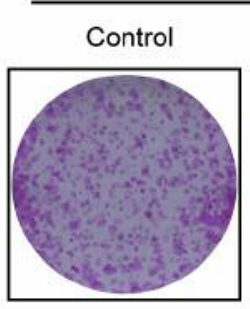

WHSC1-KD1
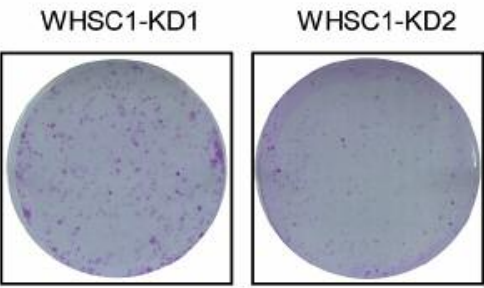

E

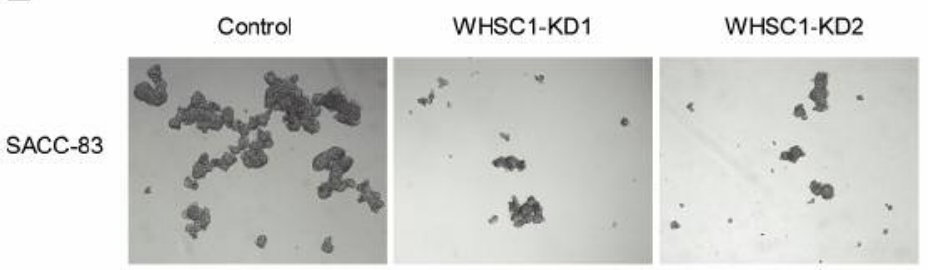

B

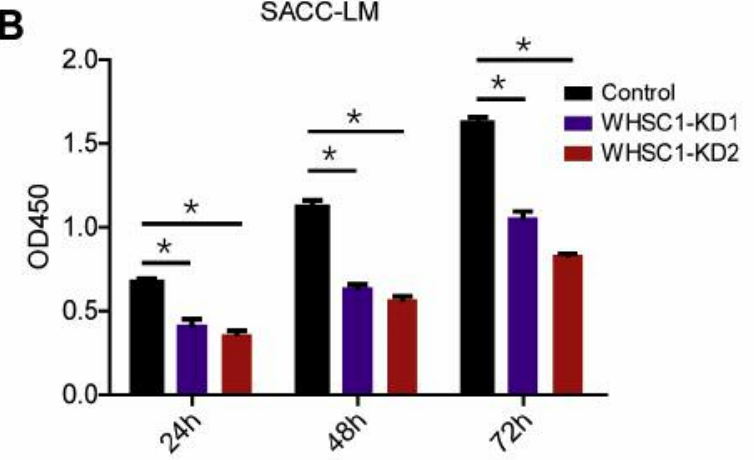

D
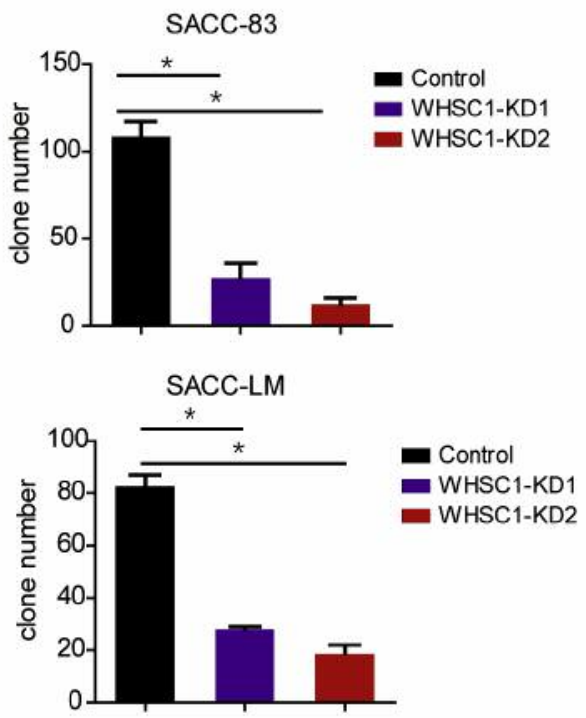

$\mathbf{F}$

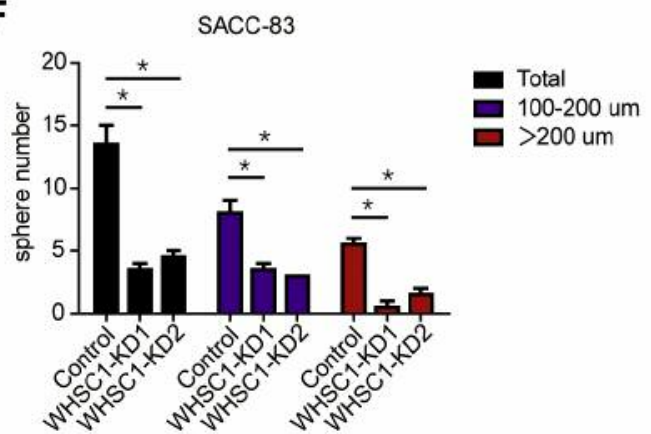

Figure 2. WHSC1 knockdown inhibits SACC cell growth and cancer stem cells. (A and B) In vitro growth of control and WHSC1-KD SACC-83 and SACC-LM cells as assessed by the CCK-8 assay. (C and D) Clone formation assay in control and WHSC1-KD cells. (E and F) Tumorsphere formation of the control and WHSC1-KD SSACC-83 and HSACC cells, ${ }^{*} p<0.05$, when compared with the control.

(4138, Cell Signaling Technology), anti-Survivin (2808, Cell Signaling Technology) or anti-GAPDH antibodies (5174S, Cell Signaling Technology). After washing twice with Tris buffered saline, the membranes were incubated with appropriate secondary antibody conjugated with HRP for $2 \mathrm{~h}$ at room temperature. Analysis of electrochemiluminescence was performed according to the manufacturer's instructions (WBKLS0050, Millipore).
In vivo study. BALB/C male nude mice, aged 4-6 weeks and weighing 20-25 g, were purchased from SHANGHAI SLAC (Shanghai, P.R. China). All mice were maintained in a HEPAfiltered environment at $24-25^{\circ} \mathrm{C}$ and humidity was maintained at 50 $60 \%$. All animal experiments were approved by the Institutional Animal Care and Use Committee and performed in compliance with the NIH Guide. 
A

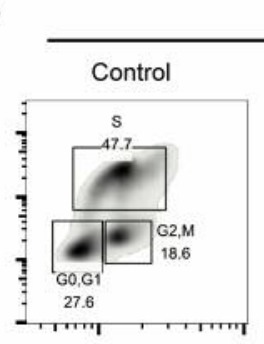

SACC-83

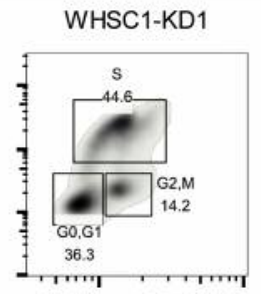

SACC-LM
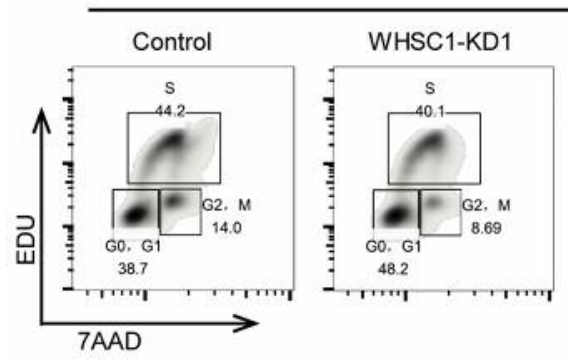
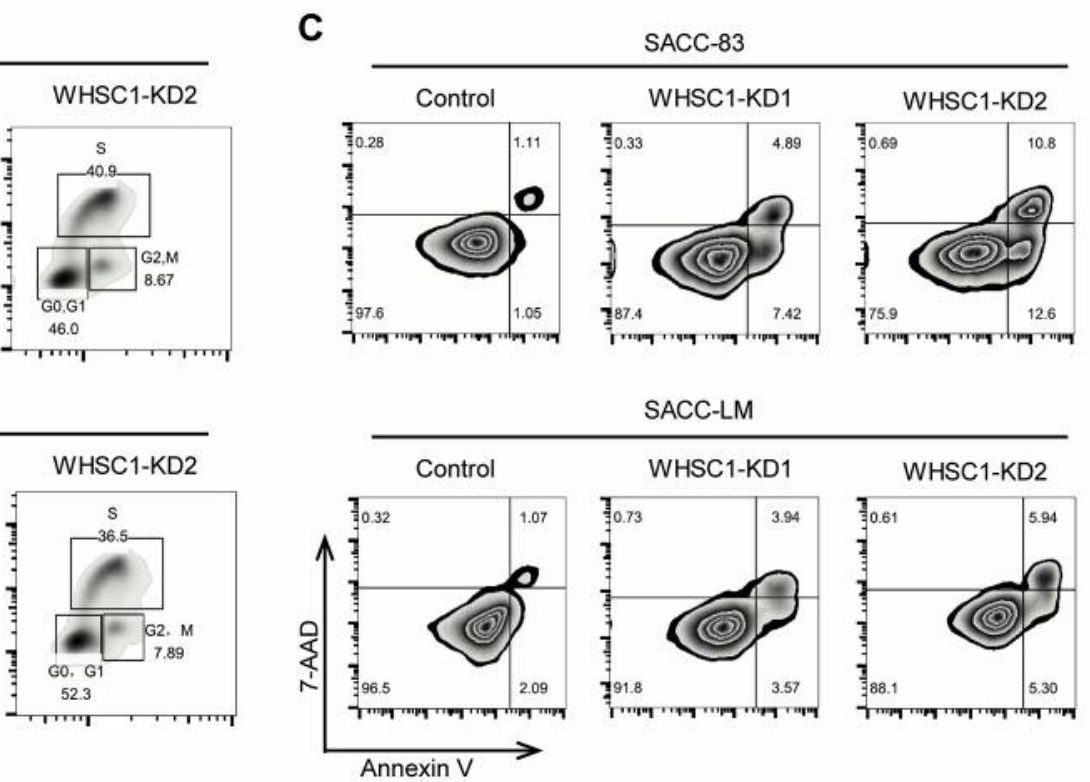

SACC-83
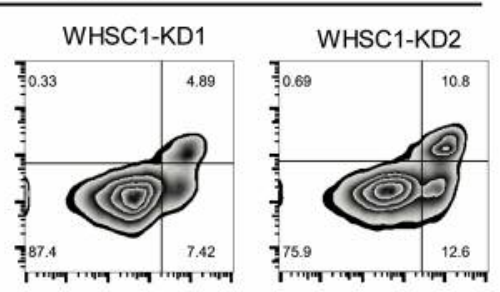

SACC-LM
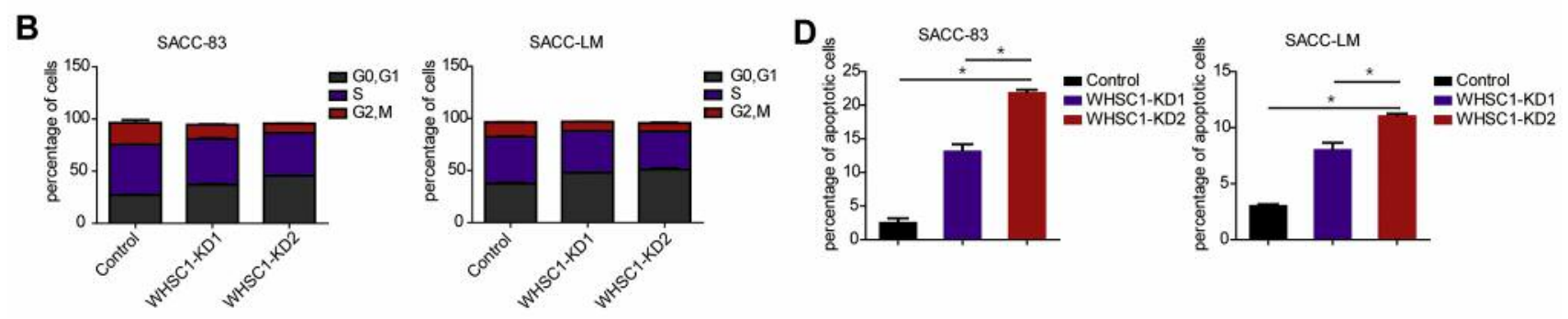

Figure 3. Effect of WHSC1 knockdown on cell cycle and apoptosis. (A and B) Control and WHSC1-KD SACC-83 and SACC-LM cells were incubated with 5-ethynyl-2 deoxyuridine (EdU) for two hours and stained with anti-EDU antibody and 7AAD. (C and D) Cells were stained with Annexin $V$ and $7 A A D$, and analyzed by flow cytometry. * $<<0.05$, when compared with the control.

Xenograft mouse models of salivary adenoid cystic carcinoma were established by subcutaneously injecting $1 \times 10^{6}$ transfected SACC cells in the flank of nude mice. Tumor growth was measured weekly with calipers. Tumor volume was calculated using the formula $\left(\mathrm{L} \times \mathrm{W}^{2}\right) \times 1 / 2$, where $\mathrm{W}$ and $\mathrm{L}$ represent the perpendicular minor and major dimension, respectively. All animals were sacrificed 21 days after tumor cell implantation. At autopsy the tumor was removed and weighed.

ChIP-qPCR analysis. $2 \times 10^{7}$ SACC- 83 and WHSC1-knockdown SACC-83 cells were crosslinked, lysed and sheared to about 200700 DNA base pairs in length using UCD-300 (Bioruptor, BE). ChIP was performed using Magnetic ChIP kit according to manufacturer's instructions (17-371, Millipore). Quantification of ChIP-enriched DNA was then performed by qPCR. The ChIP antibodies used were anti-H3K36me2 (61019, Active Motif, CA, USA) and normal rabbit IgG. Primers used are listed in Table II.

Statistical analysis. All experiments were repeated at least three times as indicated. Data are expressed as mean \pm standard deviation. All statistical analyses were performed using SPSS16.0 software (SPSS inc., Chicago, IL, USA). Data comparisons between two groups were performed using the Student's $t$-test. A value of $p<0.05$ was regarded as statistically significant.

\section{Results}

WHSC1 expression is upregulated in human SACC. To assess WHSC1 expression in human SACC specimens, GSE59701 datasets were obtained from the GEO website and a list of expression of 20,201 genes from 12 SACC samples with matched normal tissues was recorded. WHSC1 mRNA expression was significantly higher in SACC tissues compared with paired normal controls $(p<0.01)$ (Figure 1A). WHSC1 expression was further investigated in SACC specimens. RT-qPCR analysis showed that WHSC1 mRNA expression was also significantly higher in 23 SACC patients' biopsies compared with their paracarcinoma normal salivary gland specimens $(p<0.01)$ (Figure 1B). Immunohistochemical analyses further revealed significantly higher WHSC1 expression in 23 SACC patients' biopsies compared with 

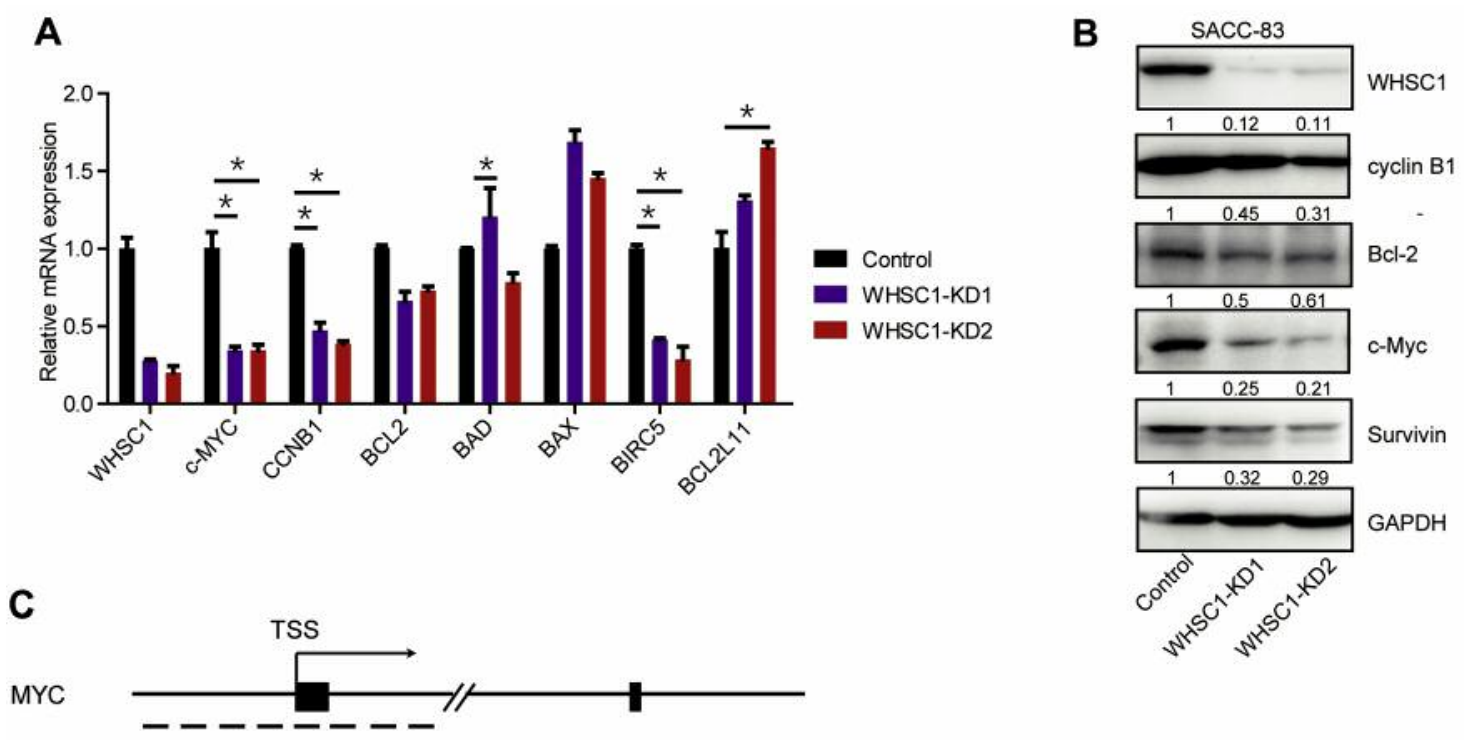

p1 p2 p3 p4 p5 p6 p7 p8
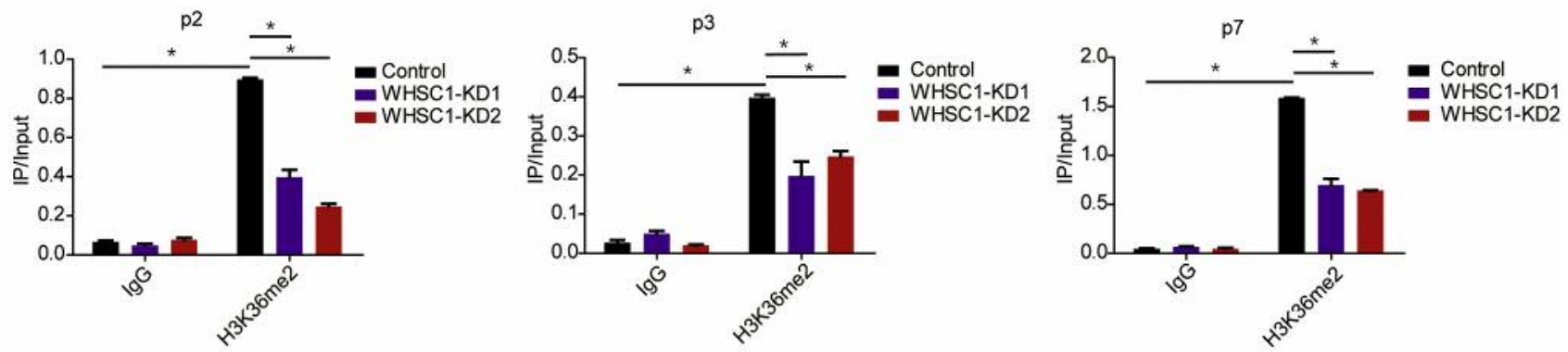

Figure 4. WHSC1 regulates cell apoptosis- and stemness-related genes by mediating H3K36me2 modification. (A) RT-qPCR of stemness-, tumor growth and apoptosis-associated genes in control and WHSC1-KD SACC-83 cells. (B) Western blot analysis of indicated proteins in control and WHSC1-KD SACC-83 cells. GAPDH served as a loading control. (C) ChIP-qPCR analysis of H3K36me2 enrichment in the MYC gene promoter in control and WHSC1-KD SACC-83 cells. *p<0.05, when compared to the control.

their paracarcinoma normal salivary gland specimens $(p<0.05)$ (Figure 1C and D).

WHSCl knockdown inhibits SACC cell growth and cancer stem cells. Knockdown of WHSC1 in SACC-83 and SACCLM cells was performed by transfecting them with WHSC1specific short hairpin RNA. The effect of WHSC1 knockdown on cell growth was assessed by examining cell proliferation as well as clone and tumorsphere formation. The SACC-83 and SACC-LM cells transfected with WHSC1-KD1 or WHSC1-KD2 showed significantly reduced cell proliferation compared to SACC-83 and SACC-LM cell controls $(p<0.05)$ (Figure 2A and B). Clone formation was significantly inhibited in the SACC-83 and SACC-LM cells transfected with WHSC1-KD1 or WHSC1-KD2 compared to SACC-83 and SACC-LM cell control $(p<0.05)$ (Figure 2C and 2D).
Cancer stem cells (CSCs) are closely correlated with tumorigenesis in many solid tumors $(14,15)$. Intriguingly, WHSC1 knockdown markedly reduce the ability of SACC cells to form tumor spheres (Figure 2E and F), suggesting that WHSC1 may affect cancer stem cell properties in SACC.

Effect of WHSC1 knockdown on the cell cycle. The effect of WHSC1-knockdown on the cell cycle was assessed by the Edu assay. As shown in Figure 3A and B, cell distribution at phase $S$ and $G_{2} / M$ was significantly reduced in the SACC83 and SACC-LM cells transfected with WHSC1-KD1 or WHSC1-KD2 compared to SACC-83 and SACC-LM cell control $(p<0.05)$.

WHSC1 regulates cell apoptosis- and stemness-related genes. The effects of WHSC1-knockdown on cell apoptosis were assessed by the Annexin V-7AAD assay. WHSC1 
A

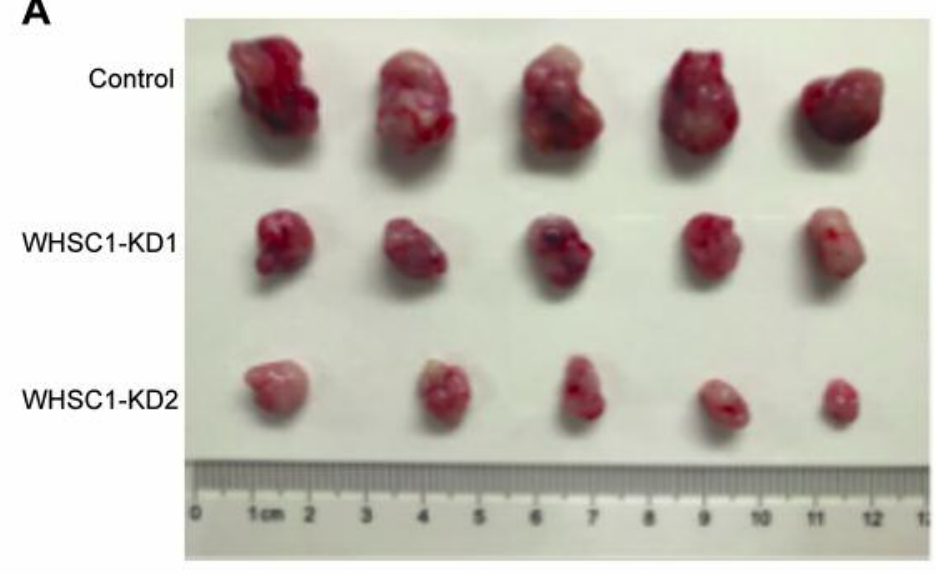

C

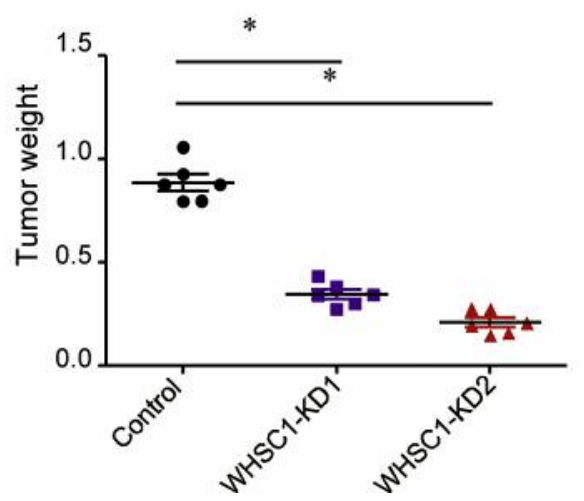

D

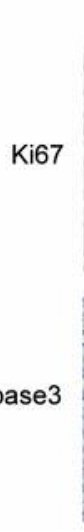

B

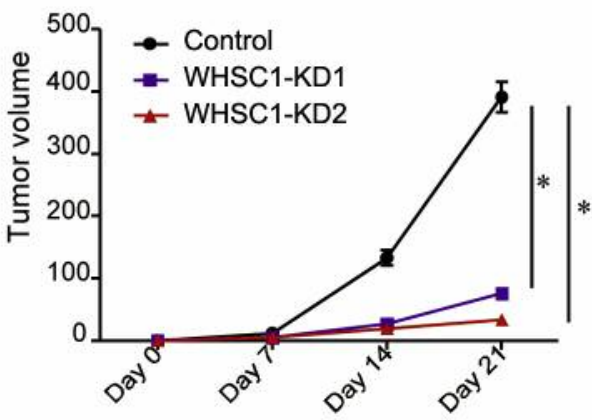

Control

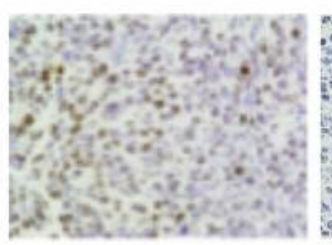

WHSC1-KD1

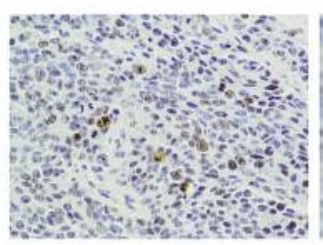

WHSC1-KD2
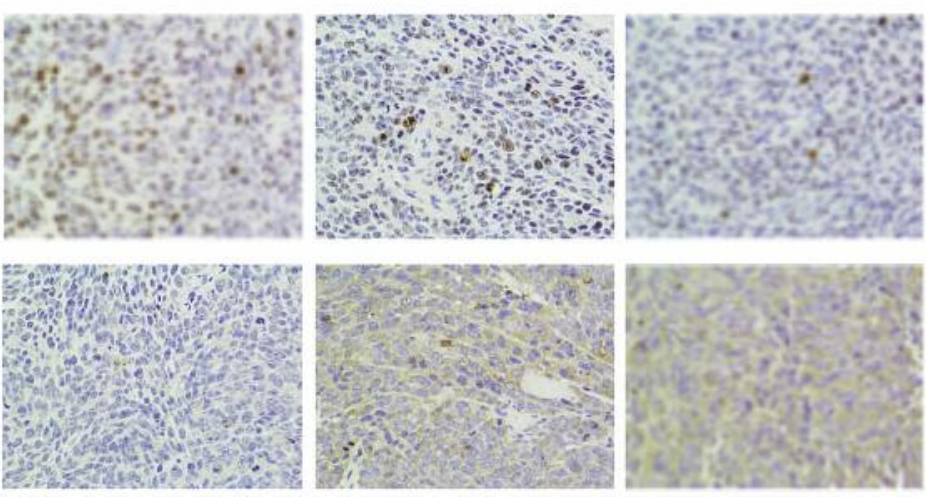

Figure 5. WHSC1 knockdown inhibits SACC progression in vivo. (A) Macroscopic image of tumor size (upper panel) and histogram showing the volume change at indicated time points and tumor weights at day 21 when mice were sacrificed. (B) Tumor size was evaluated on the indicated days, tumor volume was calculated using the formula $V=0.5^{*} a^{*} b^{*} b$ (a represent the long diameter and $b$ for short diameter). (C) Tumor weight was measured when mice were sacrificed at Day 21.(D) IHC staining of cleaved-caspase 3 and Ki67 in representative tumor tissues of control and WHSC1-KD groups. ${ }^{*} p<0.05$, when compared to the control.

knockdown in SACC-83 and SACC-LM cells significantly increased cell apoptosis compared to SACC-83 and SACCLM cell control $(p<0.05)$ (Figure 3C and D).

c-Myc, a tumor stem cell differentiation-related factor, was markedly down-regulated in the SACC-83 and SACC-LM cells transfected with WHSC1-KD1 or WHSC1-KD2 compared to the control $(p<0.05)$ (Figure 4A and B). Survivin and Bcl-2, two anti-apoptotic factors $(16,17)$, were significantly inhibited in the SACC-83 and SACC-LM cells transfected with WHSC1-KD1 or WHSC1-KD2 compared to the control $(p<0.05)$ (Figure 4B). Furthermore, cyclin B1, which is related to $G_{2} / M$ checkpoint (18), was also reduced in the SACC-83 and SACC-LM cells transfected with WHSC1-KD1 or WHSC1-KD2 compared to the control $(p<0.05)$ (Figure 4B). These results indicate that WHSC1 knockdown downregulates the expression of apoptotic and stemness-related genes in SACC cells.
WHSC1 mediates H3K36me2 modification in MYC loci. WHSC1 is an H3K36me2 histone methyltransferase. H3K36 methylation promotes gene transcription. ChIP-qPCR analysis demonstrated that WHSC1 knockdown reduced H3K36me2 modification in the MYC gene promotor (Figure 4C). These finding indicated that the depletion of WHSC1 may lead to more condensed chromatin in the MYC gene locus and thereby inhibit the transcription of c-Myc in SACC cells. Therefore, WHSC1 may directly regulate c-Myc expression by mediating H3K36me2 modification.

WHSCl knockdown inhibits SACC tumorigenesis in vivo. The effects of WHSC1-knockdown on tumorigenesis were evaluated in a mouse model of SACC. The animals, with the tumor derived from the SACC-83 and SACC-LM cells transfected with WHSC1-KD1 or WHSC1-KD2, showed significantly reduced tumor size and weight as compared to 
the control $(p<0.05)$ (Figure 5A-C). Cleaved caspase 3 positive cells were significantly increased in the SACC-83 and SACC-LM cells transfected with WHSC1-KD1 or WHSC1-KD2 compared to the control $(p<0.05)$. However, Ki67 expression was similar in all groups (Figure 5D). These results demonstrate that WHSC1 knockdown suppresses tumorigenesis and enhances apoptosis in vivo.

\section{Discussion}

In the present study, WHSC1 was found to be up-regulated in human SACC, and WHSC1 upregulation inhibited SACC cell line apoptosis both in vitro and in vivo. WHSC1 regulated transcription of key intracellular signaling molecules including BCL-2 and SOX2, by mediating their H3K36me2 modification.

It is shown, in this report, that high levels of WHSC1 in SACC cells promoted proliferation and inhibited apoptosis through epigenetically reprograming a subset of genes, indicating the MYC gene. WHSC1 knockdown significantly reduced $\mathrm{H} 3 \mathrm{~K} 36 \mathrm{me} 2$ modifications in the MYC promoter region, likely leading to more condensed chromatin and reduced MYC transcription.

Our results suggest a close relationship between the epigenetic regulator, WHSC1, and key intracellular transcription factors and molecules. MYC is a key stemness-related gene and an oncogenic driver that promotes malignant transformation of many tumors $(19,20)$. WHSC1 knockdown significantly decreased the H3K36me2 modification level in MYC gene locus, leading to inhibition of transcription of this key oncogene.

In conclusion, WHSC1 expression was up-regulated in human SACC patients. Knockdown of WHSC1 in SACC cell lines was associated with decreased cell proliferation, increased apoptosis and decreased tumorigenesis. WHSC1 may be a potential target for effective therapy of SACC.

\section{Acknowledgements}

This study is financially supported by the grants of National Natural Science Foundation of China (Nos. 81771087 and 81470755), and Shandong Provincial Natural Science Foundation, China (No. ZR2017BH005).

\section{Conflicts of Interest}

The Authors declare no conflicts of interest regarding this study.

\section{Authors' Contributions}

CHAO LIU and JIA-WEI ZHENG designed the study, analyzed the data, prepared the figures, and wrote draft manuscript; CHAO LIU and ZE-LIANG ZHAO performed in vitro study; HAI-WEI WU and LING ZHANG performed in vivo study; ZHIJIAN YANG and ROBERT M. HOFFMAN oversaw the study and revised the manuscript.

\section{References}

1 Tian Z, Li L, Wang L, Hu Y and Li J: Salivary gland neoplasms in oral and maxillofacial regions: a 23-year retrospective study of 6982 cases in an eastern Chinese population. Int J Oral Maxillofac Surg 39: 235-242, 2010. PMID: 19951834, DOI: 10.1016/j.ijom.2009.10.016

2 Renehan A, Gleave EN, Hancock BD, Smith P and McGurk M: Long-term follow-up of over 1000 patients with salivary gland tumours treated in a single centre. Br J Surg 83: 1750-1754, 1996. PMID: 9038559

3 Anderson JN, Jr., Beenken SW, Crowe R, Soong SJ, Peters G, Maddox WA and Urist MM: Prognostic factors in minor salivary gland cancer. Head Neck 17: 480-486, 1995. PMID: 8847206

4 Laurie SA, Ho AL, Fury MG, Sherman E and Pfister DG: Systemic therapy in the management of metastatic or locally recurrent adenoid cystic carcinoma of the salivary glands: a systematic review. Lancet Oncol 12: 815-824, 2011. PMID: 21147032, DOI: 10.1016/S1470-2045(10)70245-X

5 Kokemueller H, Eckardt A, Brachvogel P and Hausamen JE: Adenoid cystic carcinoma of the head and neck - a 20 years experience. Int J Oral Maxillofac Surg 33: 25-31, 2004. PMID: 14690656, DOI: 10.1054/ijom.2003.0448

6 Ho AS, Kannan K, Roy DM, Morris LG, Ganly I, Katabi N, Ramaswami D, Walsh LA, Eng S, Huse JT, Zhang J, Dolgalev I, Huberman K, Heguy A, Viale A, Drobnjak M, Leversha MA, Rice CE, Singh B, Iyer NG, CLeemans CR, Bloemena E, Ferris RL, Seethala RR, Gross BE, Liang Y, Sinha R, Peng L, Raphael BJ, Turcan S, Gong Y, Schultz N, Kim S, Chiosea S, Shah JP, Sander C, Lee W and Chan TA: The mutational landscape of adenoid cystic carcinoma. Nat Genet 45: 791-798, 2013. PMID: 23685749, DOI: $10.1038 /$ ng.2643

7 West RB, Kong C, Clarke N, Gilks T, Lipsick JS, Cao H, Kwok S, Montgomery KD, Varma S and Le QT: MYB expression and translocation in adenoid cystic carcinomas and other salivary gland tumors with clinicopathologic correlation. Am J Surg Pathol 35: 92-99, 2011. PMID: 21164292, DOI:10.1097/ PAS.0b013e3182002777

8 Kuo AJ, Cheung P, Chen K, Zee BM, Kioi M, Lauring J, Xi Y, Park BH, Shi X Garcia BA, Li W and Gozani O: NSD2 links dimethylation of histone $\mathrm{H} 3$ at lysine 36 to oncogenic programming. Mol Cell 44: 609-620, 2011. PMID: 22099308, DOI: $10.1016 /$ j.molcel.2011.08.042

9 Santra M, Zhan F, Tian E, Barlogie B and Shaughnessy J Jr.: A subset of multiple myeloma harboring the $\mathrm{t}(4 ; 14)(\mathrm{p} 16 ; \mathrm{q} 32)$ translocation lacks FGFR3 expression but maintains an IGH/MMSET fusion transcript. Blood 101: 2374-2376, 2003. PMID: 12433679 DOI: 10.1182/blood-2002-09-2801

10 Keats JJ, Maxwell CA, Taylor BJ, Hendzel MJ, Chesi M, Bergsagel PL, Larratt LM, Mant MJ, Reiman T, Belch AR and Pilarski LM: Overexpression of transcripts originating from the MMSET locus characterizes all $\mathrm{t}(4 ; 14)(\mathrm{p} 16 ; \mathrm{q} 32)$-positive multiple myeloma patients. Blood 105: 4060-4069, 2005. PMID: 15677557, DOI: 10.1182/blood-2004-09-3704

11 Yang P, Guo L, Duan ZJ, Tepper CG, Xue L, Chen X, Kung HJ, Gao AC, Zou JX and Chen HW: Histone methyltransferase NSD2/MMSET mediates constitutive NF-kappaB signaling for cancer cell proliferation, survival, and tumor growth via a feedforward loop. Mol Cell Biol 32: 3121-3131, 2012. PMID: 22645312, DOI: 10.1128/MCB.00204-12 
12 Ezponda T, Popovic R, Shah MY, Martinez-Garcia E, Zheng Y, Min DJ, Will C, Neri A, Kelleher NL, Yu J and Licht JD: The histone methyltransferase MMSET/WHSC1 activates TWIST1 to promote an epithelial-mesenchymal transition and invasive properties of prostate cancer. Oncogene 32: 2882-2890, 2013. PMID: 22797064, DOI: 10.1038/onc.2012.297

13 Yuan H, Li N, Fu D, Ren J, Hui J, Peng J, Liu Y, Qiu T, Jiang M, Pan Q, Han Y, Wang X, Li Q and Qin J: Histone methyltransferase SETD2 modulates alternative splicing to inhibit intestinal tumorigenesis. J Clin Invest 127: 3375-3391, 2017. PMID: 28825595, DOI: $10.1172 / J C I 94292$

14 Patrawala L, Calhoun T, Schneider-Broussard R, Zhou J, Claypool K and Tang DG: Side population is enriched in tumorigenic, stem-like cancer cells, whereas ABCG2+ and ABCG2- cancer cells are similarly tumorigenic. Cancer Res 65: 6207-6219, 2005. PMID:16024622, DOI:10.1158/00085472.CAN-05-0592

15 Britton KM, Eyre R, Harvey IJ, Stemke-Hale K, Browell D, Lennard TW and Meeson AP: Breast cancer, side population cells and ABCG2 expression. Cancer Lett 323: 97-105, 2012. PMID: 22521545, DOI:10.1016/j.canlet.2012.03.041

16 Reed JC: Bcl-2 on the brink of breakthroughs in cancer treatment. Cell Death Differ 25: 3-6, 2018. PMID: 29227986, DOI: $10.1038 / \mathrm{cdd} .2017 .188$

17 Shojaei F, Yazdani-Nafchi F, Banitalebi-Dehkordi M, Chehelgerdi $\mathrm{M}$ and Khorramian-Ghahfarokhi M: Trace of survivin in cancer. Eur J Cancer Prev, 2018. PMID: 29847456, DOI: 10.1097/ CEJ.0000000000000453
18 Zhang LL, Feng ZL, Su MX, Jiang XM, Chen X, Wang Y, Li A, Lin LG and Lu JJ: Downregulation of Cyclin B1 mediates nagilactone E-induced $\mathrm{G}_{2}$ phase cell cycle arrest in non-small cell lung cancer cells. Eur J Pharmacol 830: 17-25, 2018. PMID: 29680228, DOI: 10.1016/j.ejphar.2018.04.020

19 Van Schaijik B, Davis PF, Wickremesekera AC, Tan ST and Itinteang T: Subcellular localisation of the stem cell markers OCT4, SOX2, NANOG, KLF4 and c-MYC in cancer: a review. J Clin Pathol 71: 88-91, 2018. PMID: 29180509, DOI: 10.1136/jclinpath-2017-204815

20 Bahr C, Von Paleske L, Uslu VV, Remeseiro S, Takayama N, Ng SW, Murison A, Langenfeld K, Petretich M, Scognamiglio R, Zeisberger P, Benk AS, Amit I, Zandstra PW, Lupien M, Dick JE, Trumpp A and Spitz F: A Myc enhancer cluster regulates normal and leukaemic haematopoietic stem cell hierarchies. Nature 553: 515-520, 2018. PMID: 29342133, DOI: 10.1038/nature25193

Received January 19, 2019

Revised February 4, 2019

Accepted February 5, 2019 